\title{
Type 1 medication review based on a pharmacy's electronic medication records: first steps towards an algorithm to stratify patients for tailored pharmacy services
}

\author{
Revisão terapêutica tipo 1 com base nos registos eletrónicos da farmácia: primeiros passos para o \\ desenho de um algoritmo que permite estratificar doentes para a prestação de serviços de farmácia \\ personalizados
}

\author{
Ligia Reis ${ }^{1,2}$, Miguel Monteiro ${ }^{2}$, Luis Lourenço ${ }^{1}$, João Gregório ${ }^{1 *}$ \\ ${ }^{1}$ CBIOS - Universidade Lusófona's Research Center for Biosciences \& Health Technologies, Campo Grande 376, 1749-024 \\ Lisboa, Portugal \\ ${ }^{2}$ Farmácia Central do Cacém, Praceta Aquilino Ribeiro, 5/6 - r/c, 2735-060 Agualva-Cacém, Portugal
}

*corresponding author: joao.gregorio@ulusofona.pt

\begin{abstract}
Algorithms, queries, and knowledge-based systems are among approaches to screen electronic patient records stored in databases and support pharmacist medication reviews. The aim of this study was to perform a type 1 medication review and identify clusters that enable the definition of an algorithm to tailor pharmacy professional interv A retrospective observational study was conducted on a convenience sample of pharmacy records. Records were included if patients had a medication dispensing history between June 2017 - July 2018 and used two or more chronic medications. Statistical analysis used a two-step cluster to identify common characteristics among fifty-five sets of patient records which underwent Type 1 medication review. The median number of drugs used per patient was five [IQR: 3.0 - 7.0]. 18.2\% of patients had inappropriate drugs, and $30.9 \%$ had moderate or major interaction potential. Four clusters were identified based on the variables of interactions, number of drugs used, contraindications, Beers criteria and measurable biomarkers, allowing to envision possible pharmaceutical interventions, as well as the priority in providing that intervention. The identification of patient clusters via medication review of electronic records of pharmacy patients supports the design of criteria-based algorithms, likely to be automated.
\end{abstract}

Keywords: Pharmaceutical services; medication review; electronic health records; algorithms

\begin{abstract}
Resumo
Algoritmos, queries e sistemas baseados em conhecimento são algumas abordagens que permitem ao realizar revisão terapêutica do tipo 1 aos registos eletrónicos de doentes (RED). O objetivo deste estudo foi realizar uma revisão terapêutica do tipo 1 numa amostra de RED de uma farmácia comunitária, permitindo a identificação de clusters que sinalizem os doentes que requerem intervenções farmacêuticas. Estudo observacional retrospectivo numa amostra de conveniência de RED. Foram incluídos os RED com histórico de dispensa entre junho de 2017 e julho de 2018 e que apresentavam 2 ou mais medicamentos crónicos. Utilizou-se o método de análise de clusters em duas fases para identificar características comuns entre os doentes. Cinquenta e cinco RED foram submetidos à revisão terapêutica tipo 1. A mediana do número de medicamentos usados foi de cinco [IQR: 3,0 - 7,0]. 18,2\% dos doentes usavam medicamentos inapropriados e 30,9\% possuíam potencial moderado ou grave de interação entre medicamentos. Foram identificados quatro clusters, aos quais foram atribuídas as intervenções farmacêuticas consideradas mais adequadas, bem como a prioridade da sua prestação. A revisão terapêutica aplicada aos RED permitiu a identificação de clusters de doentes, o que, por sua vez, permitirá o desenho de um algoritmo baseado em critérios com possibilidade de ser automatizado.
\end{abstract}

Palavras-chave: Serviços farmacêuticos; revisão terapêutica; registos eletrónicos medicamentos; algoritmos 


\section{Introduction}

As the burden of Non-Communicable Diseases (NCD) increases globally, chronic disease management has become central to every health system (1). Among several models to deal with this threat, the Chronic Care Model has emerged as an interprofessional collaboration model suited to the challenges brought by NCD's high burden on health systems (2). This model has in turn provided the context to the rethinking of health professionals' roles and duties (3). Community pharmacists have joined this movement after the emergence of pharmaceutical care concepts and the implementation of several projects that showcased community pharmacists as effective primary care professionals (4).

Since the 1990s, medication reviews were included in community pharmacy practice. Pharmacist-led medication review services are available in Europe (UK, NL, BE, SE, DK), United States of America (USA), Australia, Canada and New Zealand $(5,6)$. The Pharmaceutical Care Network Europe (PCNE) defines medication review as "a structured evaluation of patients' medications with the aim of optimizing medication use and improving health outcomes". This entails detecting drug related problems (DRPs) and recommending interventions (7). Four types of review are identified depending on the patient information available to the pharmacist (Table 1). All types of Medication reviews are always constituted by a diagnostic intervention that aims to identify DRPs. All but type 1 require the patient to be present at the point of care, both to gather important information and to provide an educational intervention to support patient knowledge and adherence (8).

When provided, medication reviews resulted in a decrease in the number of drug-related problems, more changes in medication, more drugs with decreased dosage, and a greater decrease or smaller increase in the number of drugs (6). The reviews also have a positive benefit on patient outcomes specifically on the attainment of clinical biomarkers goals (5), and improvement of process outcomes such as reduced polypharmacy, more appropriate formulations, and more appropriate choices of medications (8). The appropriateness of medications can also be assessed with different tools. Among them, Beers' criteria is one of the most commonly used (9), assisting healthcare providers in improving medication safety in older adults by identifying medications for which the risks of their use outweigh the benefits (10).

\section{Introdução}

À medida que a carga das doenças não transmissíveis (DNT) aumenta globalmente, a gestão das doenças crónicas torna-se central para todos os sistemas de saúde (1). Entre os vários modelos para lidar com esta ameaça, o Modelo de Cuidados Crónicos emergiu como um modelo de colaboração interprofissional adequado aos desafios trazidos pela elevada carga das DNT sobre os sistemas de saúde (2). Este modelo, por sua vez, forneceu o contexto para repensar os papéis e deveres dos profissionais de saúde (3). Os farmacêuticos comunitários aderiram a este movimento após o surgimento de conceitos de Cuidados Farmacêuticos e a implementação de vários projetos posicionaram o farmacêutico comunitário como um profissional de cuidados de saúde primários efetivo (4).

Desde a década de 1990, as revisões terapêuticas foram incluídas na prática da farmácia comunitária. O serviço de revisão terapêutica conduzido por farmacêuticos está disponível na Europa (UK, NL, BE, SE, DK), Estados Unidos da América (EUA), Austrália, Canadá e Nova Zelândia $(5,6)$. A Pharmaceutical Care Network Europe (PCNE) define a revisão terapêutica como "uma avaliação estruturada dos medicamentos dos doentes com o objetivo de otimizar o uso de medicamentos e melhorar os resultados em saúde". Isso envolve a deteção de problemas relacionados a medicamentos (PRM) e a recomendação de intervenções (7). Conhecem-se quatro tipos de revisão, dependendo das informações do doente disponíveis para o farmacêutico (Tabela 1). Todos os tipos de revisão terapêutica incluem uma intervenção diagnóstica que visa identificar PRM. Todos, exceto o tipo 1, requerem a presença do doente tanto para recolher informações importantes quanto para prestar uma intervenção educacional que promova o conhecimento e a adesão do doente (8).

Quando realizadas, as revisões terapêuticas resultam numa diminuição do número de PRM, ajustes à medicação, diminuição de doses e uma diminuição ou ligeiro aumento do número de medicamentos utilizado pelo utente (6). Evidenciam ainda um benefício positivo nos resultados, especificamente nos biomarcadores clínicos (5), e na melhoria dos resultados do processo, como redução da polimedicação, adequação da formulação e escolha mais adequada de medicamento (8). Neste processo a avaliação de medicamentos potencialmente inapropriados pode ser realizada com diferentes ferramentas. Entre estas, o critério de Beers é uma das mais utilizadas (9), auxiliando os profissionais de saúde a promover a segurança dos medicamentos 
Table 1 - Types of medication review (PCNE 2012)

Tabela 1 - Tipos de revisão terapêutica (PCNE 2012)

\begin{tabular}{|l|l|}
\hline TYPE & DESCRIPTION \\
\hline $\begin{array}{l}\text { Type 1 - Simple medication } \\
\text { review }\end{array}$ & $\begin{array}{l}\text { Based on the available medication history (electronic medication record) in the pharmacy, this } \\
\text { non-clinical review addresses simple issues relating to the medication with the purpose of } \\
\text { highlighting more complex issues for other type of review or services. Useful to reveal drug } \\
\text { interactions, some side-effects, unusual dosages and some adherence issues. }\end{array}$ \\
\hline $\begin{array}{l}\text { Type 2A - Intermediate } \\
\text { medication review }\end{array}$ & $\begin{array}{l}\text { Performed when the patient can be approached for information, this review is based on medication } \\
\text { history and patient information. Helps to detect drug interactions, some side-effects, unusual } \\
\text { dosages, adherence issues, drug-food interactions, effectiveness issues, side effects, and problems } \\
\text { with over-the-counter (OTC) medicines. }\end{array}$ \\
\hline $\begin{array}{l}\text { Type 2B - Intermediate } \\
\text { medication review }\end{array}$ & $\begin{array}{l}\text { Performed if medical information is also available, this review is based on medication history and } \\
\text { medical information. It reveals drug interactions, some side-effects, unusual dosages, adherence } \\
\text { issues, drug-food interactions, effectiveness issues, indication without a drug and drugs without } \\
\text { indication. }\end{array}$ \\
\hline $\begin{array}{l}\text { Type 3 - Advanced } \\
\text { medication review }\end{array}$ & $\begin{array}{l}\text { Based on medication history, patient information, and clinical information. Enables the } \\
\text { identification of drug interactions, some side-effects, unusual dosages adherence issues, drug-food } \\
\text { interactions, effectiveness issues, side effects, problems with OTC, indication without a drug and } \\
\text { drugs without indication, dosage issues. }\end{array}$ \\
\hline
\end{tabular}

\begin{tabular}{|l|l|}
\hline TIPO & DESCRIÇÃO \\
\hline Tipo 1 - revisão simples & $\begin{array}{l}\text { Baseada no histórico de medicamentos disponíveis na farmácia (registo eletrónico de } \\
\text { medicamentos), esta revisão, não clínica, aborda questões simples relacionadas com a terapêutica } \\
\text { com o objetivo de destacar questões mais complexas para outro tipo de revisão ou serviço. Útil } \\
\text { para revelar interações medicamentosas, algumas reações adversas, doses incomuns e alguns } \\
\text { problemas de adesão. }\end{array}$ \\
\hline Tipo 2A - revisão & $\begin{array}{l}\text { Realizada quando o doente pode ser abordado para obter informações, esta revisão é baseada no } \\
\text { histórico de terapêutica e nas informações do doente. Ajuda a detetar interações medicamentosas, } \\
\text { algumas reações adversas, doses incomuns, problemas de adesão, interações com alimentos, } \\
\text { problemas de eficácia, efeitos colaterais e problemas com medicamentos não sujeitos a receita } \\
\text { médica. }\end{array}$ \\
\hline Tipo 2B - revisão intermédia & $\begin{array}{l}\text { Realizada quando a informação médica também está disponível; esta revisão é baseada no } \\
\text { histórico de medicamentos e na informação clínica. Identifica interações medicamentosas, algumas } \\
\text { reações adversas, doses incomuns, problemas de adesão, interações com alimentos, problemas de } \\
\text { eficácia, necessidade adicional de terapêutica e medicação desnecessária. }\end{array}$ \\
\hline Tipo 3 - revisão avançada & $\begin{array}{l}\text { Com base no histórico de medicamentos, informação do doente e informação clínicas. Permite a } \\
\text { identificação de interações medicamentosas, problemas de adesão, doses incomuns, interações } \\
\text { com alimentos, problemas de eficácia, reações adversas, problemas com medicamentos não } \\
\text { sujeitos a receita médica, necessidade adicional de terapêutica e medicação desnecessária, } \\
\text { problemas de dose. }\end{array}$ \\
\hline
\end{tabular}

In spite of being a service that can enhance the pharmacists' role in the health system, the implementation of medication reviews has not been thoroughly achieved. Major barriers to implement an effective medication review service cited in the literature have been lack of time, collaboration with physicians, pharmacists' self-confidence, and patient inclusion $(11,12)$. Therefore, there is a need to enhance the efficiency of medication review services. The use of IT may unlock this potential efficiency (13), allowing more time to augment medication review provision and the development of new services. nos idosos, através da identificação de medicamentos para os quais os riscos do seu uso superam os benefícios (10).

Apesar da revisão terapêutica ser um serviço que pode enfatizar o papel do farmacêutico no sistema de saúde, a sua implementação não foi totalmente alcançada. As principais barreiras citadas na literatura para implementar um serviço eficaz de revisão terapêutica têm sido a falta de tempo, a colaboração com médicos, a baixa autoconfiança dos farmacêuticos e a inclusão de doentes $(11,12)$. Assim, torna-se necessário aumentar a eficiência do serviço de revisão terapêutica. O uso da Tecnologias da Informação pode desbloquear este 
The inception of the Digital Age in the 1960s, with the development of electronics, information technology (IT) and automated production, has shaped the practice of pharmacy (14). Nowadays, community pharmacy practice is highly computerized, collecting patient information on a continuous basis and creating a permanently updated electronic healthcare record (EHR) (15). Electronic healthcare records provide realtime access to patient information, such as the patient's medical condition, visits to healthcare providers, and images and reports of diagnostic procedures, thus enabling a complete longitudinal record of evidencebased care (15). These EHR can impact decisionmaking, how healthcare professionals and patients interact with each other, how health information is stored and used, and how patients manage their health themselves through electronic apps and devices (16).

For community pharmacists, EHRs facilitate medication reviews and the identification of drug therapy problems (16). Structured methods and appropriate software are important tools to increase pharmacist effectiveness and improve health outcomes $(17,18)$. Databases, that store patients' electronic records can be queried using search algorithms, for example, to select non-adherent patients or patients receiving specific medications (17), or to identify inpatients at risk of drug-related problems (19). Algorithms are known to support medication review processes identifying and discontinuing potentially inappropriate medications, which may otherwise lead older population to harmful events (20). Algorithms are liable to be automated and therefore able to support a continuous, reproducible, and less time-consuming medication review (21).

However, the definition of an algorithm tailored to the Portuguese pharmacies' information system is lacking. In order to design an algorithm, criteria must be defined based on the variables available on patient information of electronic records. Therefore, the aim of this study was to apply a type 1 medication review to a sample of patients' electronic medication records of a community pharmacy. This medication review will then enable the identification of clusters of patients in need of additional pharmacy professional interventions, a first step toward the design of a new decision algorithm. potencial de eficiência (13), proporcionando ganhos de tempo com aumento da oferta deste serviço e desenvolvimento de novos serviços.

O início da Era Digital na década de 1960, marcada pelo desenvolvimento da eletrónica, da tecnologia da informação e da produção automatizada, moldou a prática da farmácia (14). Hoje em dia, a prática da farmácia comunitária está fortemente informatizada, recolhendo informações do doente de forma contínua e criando registos eletrónicos de saúde permanentemente atualizados (15). Os registos eletrónicos de saúde permitem o acesso em tempo real aos dados do doente, como patologias, visitas a profissionais de saúde, imagens e relatórios de diagnóstico, possibilitando um registo longitudinal completo dos cuidados de saúde prestados (15). Estes registos eletrónicos de saúde podem afetar a tomada de decisão, a forma como profissionais de saúde e doentes interagem uns com os outros, como as informações de saúde são armazenadas e usadas e como os doentes gerem a sua própria saúde através de aplicativos ou dispositivos eletrónicos (16).

Para o farmacêutico comunitário, os registos eletrónicos de saúde facilitam as revisões terapêuticas e a identificação de PRM (16). Métodos estruturados e softwares apropriados são ferramentas importantes para aumentar a efetividade do farmacêutico e melhorar os resultados em saúde $(17,18)$. As bases de dados, que armazenam os registos eletrónicos de saúde, podem ser consultadas usando algoritmos de pesquisa, por exemplo, para selecionar doentes não aderentes ou doentes que recebem medicamentos específicos (17), ou para identificar doentes internados com risco de PRM (19). Os algoritmos são conhecidos por apoiar os processos de revisão terapêutica, identificando e descontinuando medicamentos potencialmente inadequados, que colocam a população idosa em risco (20). Os algoritmos são passíveis de automatização e, portanto, capazes de suportar a revisão terapêutica que se pretende contínua, reprodutível e menos demorada (21).

No entanto, falta a definição de um algoritmo adaptado ao sistema informático das farmácias portuguesas. De forma a desenhar um algoritmo, é necessário definir os critérios a utilizar com base na informação do doente disponível no registo eletrónico. Assim, o objetivo deste estudo foi aplicar uma revisão terapêutica do tipo 1 a uma amostra de registos eletrónicos de medicamentos de doentes de uma farmácia comunitária. Esta revisão terapêutica permitirá, então, a identificação de clusters de doentes que precisam de intervenções farmacêuticas adicionais, sendo este um primeiro passo para o projeto de um novo algoritmo de decisão. 


\section{Materials and Methods}

An exploratory retrospective observational study was conducted performing a type 1 medication review to electronic patient records. A convenience sample was used, extracted from the database of electronic medication records of a community pharmacy located in the district of Lisbon. The following inclusion criteria were used: records of continuous therapy in 12 months (June 2017-July 2018); use of two or more chronic prescription medications. Continuous therapy was defined has having records for medication dispensation fulfilling the expected duration of therapy without interruption.

To perform medication reviews, the criteria of necessity, effectiveness and safety of the 2nd Granada Consensus were applied (22). Drug safety was assessed with Sifarma ${ }^{\mathrm{TM}}$ software and included determination of interactions and contraindications, and respective level of severity. Degree of severity was classified as minor, moderate or major. Sifarma ${ }^{\mathrm{TM}}$ software enables to register and access patients' dispensing history, biomarkers, interactions, contraindications, and adverse reactions (23). Potentially inappropriate medications (PIM) were identified applying the Table 2 of Beers criteria 2015 (24), to patients over 65 years of age, classifying each drug as appropriate or inappropriate. This table lists potentially inappropriate medications for older adults outside the palliative care and hospice setting, including medications to avoid for many or most older adults, even in the absence of patient's diagnosis (25). These criteria were considered as not applicable for patients under 65 years old. Duplication of the same active substance or same therapeutic class was also identified. The Summary of Product Characteristics (SPC) of each medication was also consulted to identify the need for monitoring and which parameters to evaluate.

Statistical analysis of the data was performed using IBM SPSS version 22 and Microsoft Excel. Significance level was set to $5 \%$. Non-parametric tests were performed because there was no normal distribution in all continuous variables. For categorical and ordinal variables, Spearman correlations and Chi-Square tests were used. Two-step cluster analysis was performed to identify the number of possible clusters within the sample. Patients had to sign an informed consent to have their record stored at the pharmacy, where consent was given to use anonymized data for research purposes. No further consent was deemed necessary.

\section{Material e Métodos}

Este é um estudo exploratório, observacional e retrospetivo, cujo o objetivo foi realizar uma revisão terapêutica do tipo 1 em registos eletrónicos de doentes. Foi utilizada uma amostra de conveniência, extraída da base de dados de uma farmácia comunitária localizada no distrito de Lisboa. Foram utilizados os seguintes critérios de inclusão: histórico de terapêutica contínua em 12 meses (entre junho de 2017 a julho de 2018) e utilização de dois ou mais medicamentos crónicos. $\mathrm{O}$ histórico de terapêutica contínua foi definido como a existência de registos de dispensa de medicamentos que cumprem com a duração esperada da terapêutica sem qualquer interrupção.

Para realizar a revisão terapêutica, foram aplicados os critérios de necessidade, eficácia e segurança do $2^{\circ}$ Consenso de Granada (22). A segurança dos medicamentos foi avaliada com o software Sifarma ${ }^{\mathrm{TM}} \mathrm{e}$ incluiu a determinação de interações e contraindicações, e respetivo nível de gravidade. O grau de gravidade foi classificado como ligeiro, moderado ou grave. $\mathrm{O}$ software Sifarma ${ }^{\mathrm{TM}}$ permite registar e aceder ao histórico de dispensa, biomarcadores, interações, contraindicações e reações adversas dos doentes (23). Os medicamentos potencialmente inapropriados foram identificados aplicando-se a Tabela 2 dos critérios de Beers 2015 (24), para doentes com mais de 65 anos de idade classificando cada medicamento como apropriado ou inapropriado. Esta tabela lista os medicamentos potencialmente inadequados para idosos fora do ambiente de cuidados paliativos e lares, incluindo medicamentos a serem evitados para muitos ou a maioria dos idosos, mesmo na ausência de registo do diagnóstico (25). Para doentes com menos de 65 anos, esses critérios foram considerados não aplicáveis. Também foi identificada duplicação da mesma substância ativa ou mesma classe terapêutica. O Resumo das Características do Medicamento (RCM) de cada medicamento foi igualmente consultado para identificar a necessidade de monitorização de biomarcadores e que parâmetros avaliar.

A análise estatística dos dados foi realizada no IBM SPSS versão 22 e Microsoft Excel. O nível de significância foi estabelecido em 5\%. Foram realizados testes não paramétricos, pois não houve distribuição normal em todas as variáveis contínuas. Para variáveis categóricas e ordinais, foram utilizadas correlações de Spearman e testes de Qui-quadrado. A análise de clusters em duas fases foi realizada para identificar o número de clusters possíveis na amostra. Os doentes tiveram de assinar um 


\section{Results}

The final sample of electronic records eligible for type 1 medication review included 55 patients. $54.5 \%$ were female and $45.5 \%$ were male. Mean age of the sample was 65.67 years (SD 14.8) with a median of 68 years [IQR: 55.0 - 77.0]. Age had a normal distribution in women but not in men (Shapiro-Wilk; $p=0.634$ women; $p=0.017 \mathrm{men}$ ). In this sample, men were on average older than women, but the differences were not significant (Mann-Whitney; $\mathrm{p}=0.076$ ) (Table 2). The age variable was then categorized with a cut-off in the Beers criteria ( $<65$ years; $\geq 65$ years).

The results revealed that $58.1 \%$ of patients in the sample went to the pharmacy at least once a month. Differences between genders in frequency of pharmacy visits were not significant $(p=0.313)$. Despite the positive correlation with age, in this sample, differences between age groups and their visit frequency were also not significant (Qui-square; $\mathrm{p}=0.453$ ).

A total of 289 drugs were analysed. The median number of drugs used per patient was 5.0 [IQR: $3.0-$ 7.0]. As the variable "number of drugs" had no normal distribution (Shapiro-Wilk; $p<0.0001$ ), a new variable was categorized considering the median and using the numerical category most often used in polypharmacy literature -5 or more drugs (26). With this new variable, it was found that $43.6 \%$ of patients used 4 drugs or less, and $56.4 \%$ used 5 or more drugs. No differences were identified in the number of drugs between genders (Mann-Whitney; $\mathrm{p}=0.725$ ) (Table 2). Age had a positive correlation with the patient's total number of medications (Spearman's coefficient $=0.345, \mathrm{p}=$ $0.010)$.

Type 1 medication review showed that $87.3 \%$ of patients in the studied sample had at least one medical condition with measurable biomarkers, such as hypertension, diabetes, dyslipidaemia, or asthma. All participants over 65 years of age had one of these medical conditions $(\mathrm{p}=0.001)$, but having measurable biomarkers was not associated with the number of medications taken (Mann-Whitney; $\mathrm{p}=0.279$ ). Concerning 2015 Beers criteria, $18.2 \%$ of the sample population was found to termo de consentimento livre e esclarecido para que seus registos fossem armazenados na farmácia, onde foi dado consentimento para o uso de dados anónimos para fins de pesquisa. Nenhum consentimento adicional foi considerado necessário.

\section{Resultados}

A amostra final de registos eletrónicos elegíveis para revisão terapêutica do tipo 1 incluiu 55 doentes. Destes $54,5 \%$ eram mulheres e $45,5 \%$ homens. A média de idade da amostra foi de 65,67 anos (DP 14,8) com mediana de 68 anos [IQR: 55,0 - 77,0]. A idade teve distribuição normal nas mulheres, mas não nos homens (Shapiro-Wilk; $p=0,634$ mulheres; $p=0,017$ homens). Nesta amostra, os homens eram em média mais velhos do que as mulheres, mas as diferenças não foram significativas (Mann-Whitney; $p=0,076$ ) (Tabela 2). A variável idade foi então categorizada com um ponto de corte nos critérios de Beers ( $<65$ anos; $\geq 65$ anos).

Observou-se que 58,1\% dos doentes da amostra visitou a farmácia pelo menos uma vez por mês. As diferenças entre género na frequência de visitas à farmácia não foram significativas $(p=0,313)$. Apesar da correlação positiva com a idade, as diferenças entre as faixas etárias e a frequência de visitas também não foram significativas $(\mathrm{p}=0,453)$.

Um total de 289 medicamentos foi analisado. A mediana do número de medicamentos usados por cada doente foi de 5,0 [IQR: 3,0 - 7,0]. Como a variável "número de medicamentos" não teve distribuição normal (Shapiro-Wilk; $p<0,0001$ ), uma nova variável foi categorizada recorrendo à categoria numérica mais utilizada na literatura de polifarmácia - 5 ou mais medicamentos (26). Com esta nova variável, verificouse que $43,6 \%$ dos doentes usavam 4 medicamentos ou menos e $56,4 \%$ usavam 5 ou mais medicamentos. Não foram identificadas diferenças entre géneros quanto ao número de medicamentos (Mann-Whitney; $p=0,725$ ) (Tabela 2). A idade apresentou uma correlação positiva com o total de medicamentos do doente (coeficiente de Spearman $=0,345, \mathrm{p}=0,010$ )

A revisão terapêutica tipo 1 revelou que $87,3 \%$ dos doentes nesta amostra tinham pelo menos uma patologia com biomarcadores mensuráveis, como hipertensão arterial, diabetes, dislipidemia ou asma. Todos os participantes com mais de 65 anos apresentavam uma destas patologias $(p=0,001)$ mas ter biomarcadores mensuráveis não estava associado ao número de 
Table 2 - Dependent variable distribution by gender and age group

Tabela 2 - Distribuição das variáveis dependentes por sexo e grupo etário

\begin{tabular}{|l|c|c|c|c|}
\hline & \multicolumn{2}{|c|}{ Gender / Género } & \multicolumn{2}{c|}{ Age group / Grupo Etário } \\
\hline & $\begin{array}{c}\text { Female / } \\
\text { Feminino }\end{array}$ & $\begin{array}{c}\text { Male / } \\
\text { Masculino }\end{array}$ & $<65$ years / anos & $\geq 65$ years / anos \\
\hline $\mathrm{N}(\%)$ & $30(54.5 \%)$ & $25(45.5 \%)$ & $22(40.0 \%)$ & $33(60.0 \%)$ \\
\hline Mean age (SD) / Média da Idade (DP) & $62.6(14.8)$ & $69.4(12.4)$ & $51.1(8.5)$ & $75.4(6.5)$ \\
\hline $\begin{array}{l}\text { Median of medicines used (IQR) / } \\
\text { Mediana do número de medicamentos } \\
\text { usados (IQR) }\end{array}$ & $4.5(3.0-7.3)$ & $5.0(3.5-6.5)$ & $4.0(3.0-5.0)$ & $5.0(3.0-8.5)$ \\
\hline $\begin{array}{l}\text { Frequency of pharmacy visits / } \\
\text { Frequência de visitas à farmácia }\end{array}$ & $2(6.7 \%)$ & $2(8.0 \%)$ & $2(9.1 \%)$ & $2(6.1 \%)$ \\
\hline$<5$ visits per year/ visitas por ano & $12(40.0 \%)$ & $7(28.0 \%)$ & $10(45.5 \%)$ & $9(27.3 \%)$ \\
\hline 6-12 visits per year / visitas por ano & $10(33.3 \%)$ & $14(56.0 \%)$ & $7(31.8 \%)$ & $17(51.5 \%)$ \\
\hline $13-23$ visits per year / visitas por ano & $6(20.0 \%)$ & $2(8.0 \%)$ & $3(13.6 \%)$ & $5(15.2 \%)$ \\
\hline$>24$ visits per year / visitas por ano & \multicolumn{1}{|l}{} & & & \\
\hline
\end{tabular}

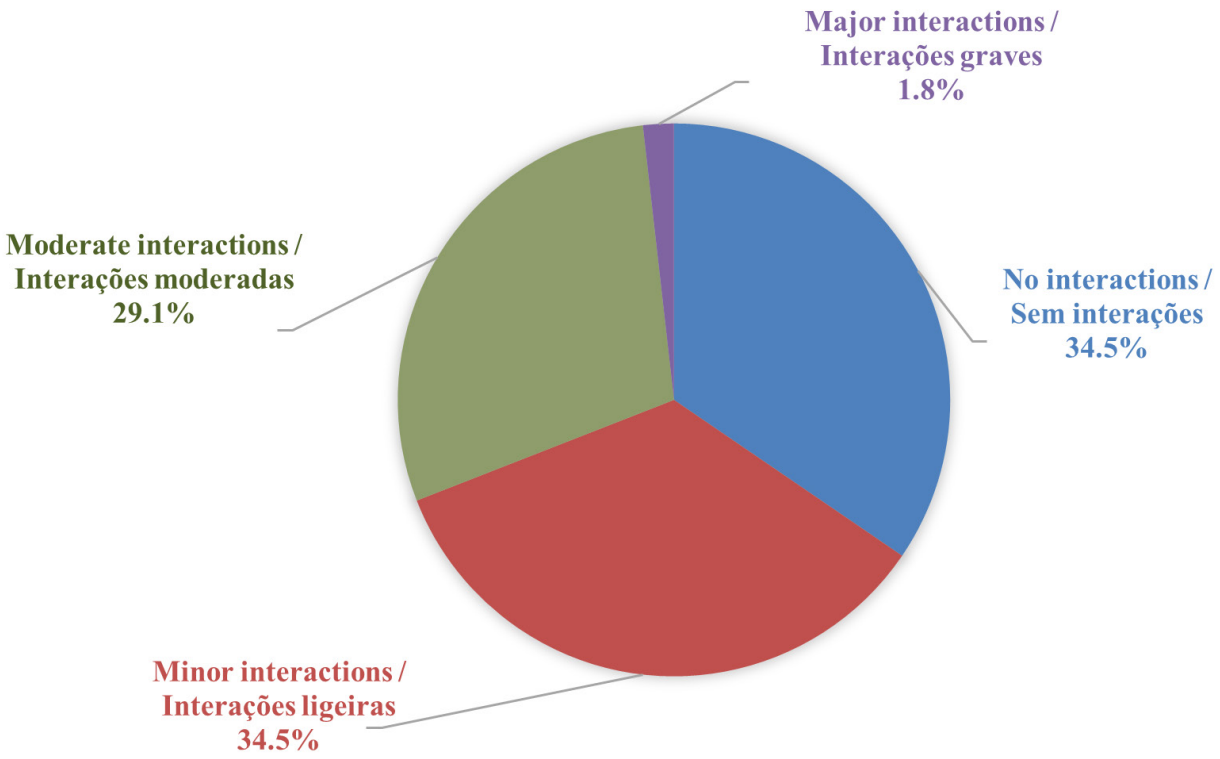

Figure 1 - Frequency of medication interactions based on level of severity $(n=55)$

Figura 1 - Frequência das interações segundo o grau de gravidade $(\mathrm{n}=55)$

take inappropriate drugs. Gender differences in Beers criteria were not significant $(\mathrm{p}=0.148)$, yet a positive correlation between the number of drugs and the Beers criteria was found (Spearman coefficient $0.322 ; \mathrm{p}=$ 0.017). When analysing drug interactions, $30.9 \%$ were found to have moderate or major interaction potential. Figure 1 shows the distribution of degrees of severity of drug interactions. No differences in the degree of severity of interaction were observed between the age groups $(p=0.388)$, between genders $(p=0.681)$, or patients with or without measurable biomarkers $(\mathrm{p}=$ medicamentos consumidos (Mann-Whitney; $p=0,279$ ).

Em relação aos critérios de Beers de 2015, identificouse que $18,2 \%$ da amostra apresentava medicamentos inapropriados. As diferenças de género nos critérios de Beers não foram significativas $(\mathrm{p}=0,148)$, mas uma correlação positiva entre o número de medicamentos e estes critérios foi encontrada (coeficiente de Spearman $0,322 ; p=0,017)$.

Ao analisar as interações medicamentosas, verificouse que $30,9 \%$ apresentavam um potencial de interação 
0.841). However, as with the Beers criteria, a positive correlation was found between the number of drugs and the severity degree of interactions (Spearman coefficient $0.671 ; p<0.001)$. The more medications one uses, the greater the likelihood of serious interactions. Another positive correlation was found between the severity of the interactions and Beers criteria (Spearman's coefficient $=0.343, \mathrm{p}=0.010$ ), which means that patients with Inappropriate Beers criteria had also a higher likelihood of using medications with moderate or major interactions.

Multicollinearity was also assessed. The highest Variance inflation factor (VIF) was 2.58 for age and number of medications. The literature considers this value to be an acceptable value, indicative of low multicollinearity. After the descriptive analysis, to uncover possible patient clusters in this sample we performed a two-step cluster analysis. Considering the statistical analysis, the variables included in the model for the two-step cluster analysis were severity degree of interactions, severity degree of contraindications, Beers criteria, number of drugs used and medical condition with measurable biomarkers.

Four clusters and one outlier patient were identified, with the characteristics presented in Table 3 . Figure 2 details the importance of the variables in the model. These clusters allowed to identify the proportion of patients who could benefit from different pharmaceutical interventions and their degree of urgency or priority.

\section{Discussion}

In this study, a type 1 medication review was performed using a sample of patients' EHRs within a community pharmacy database. This review enabled the identification of patient clusters. These clusters led us to propose different pharmaceutical interventions suitable to the perceived associated risk. For instance, two clusters ( 2 and 3 ), representing $36.3 \%$ of patients, could benefit from a type $2 \mathrm{~A}$ review, while another cluster (cluster 4) comprising $30.9 \%$ of patients would only need to measure their biomarkers to assess the moderado ou grave. A Figura 1 mostra a distribuição dos graus de gravidade das interações medicamentosas. Não foram observadas diferenças no grau de gravidade da interação entre os grupos etários $(\mathrm{p}=0,388)$, sexo $(\mathrm{p}=0,681)$ ou doentes com ou sem biomarcadores mensuráveis $(\mathrm{p}=0,841)$. Contudo, tal como nos critérios de Beers, foi encontrada uma correlação positiva entre o número de medicamentos e o grau de gravidade das interações (coeficiente de Spearman $0,671 ; \mathrm{p}<0,001)$. Quanto mais medicamentos se usa, maior a probabilidade de interações graves. Outra correlação positiva foi encontrada entre a gravidade das interações e os critérios de Beers (coeficiente de Spearman $=0,343, \mathrm{p}=0,010)$, ou seja, os doentes com medicamentos inapropriados apresentaram maior probabilidade de usar medicamentos com interações moderadas ou graves.

A multicolinearidade foi também avaliada. O maior Fator de Inflação de Variância obtido foi para a variável idade com o número de medicamentos $(\mathrm{VIF}=2,58)$. A literatura considera este um valor aceitável, indicativo de baixa multicolinearidade. Após a análise descritiva, e de forma a encontrar os possíveis clusters de doentes nesta amostra, foi realizada uma análise de cluster em duas etapas (two-step cluster analysis). As variáveis incluídas no modelo para a análise de cluster em duas etapas foram: grau de gravidade das interações, grau de gravidade das contraindicações, critérios de Beers, número de medicamentos usados e biomarcadores mensuráveis.

Foram identificados quatro clusters e um doente outlier, com as características apresentadas na Tabela 3. A Figura 2 detalha a importância das variáveis no modelo. A análise de clusters permitiu, assim, identificar a proporção de doentes que poderia beneficiar de uma intervenção farmacêutica diferente e o seu grau de urgência ou prioridade.

\section{Discussão}

Neste estudo, realizou-se uma revisão terapêutica tipo 1 usando uma amostra de registos eletrónicos de doentes de uma base de dados de farmácia comunitária. Esta revisão permitiu a identificação de clusters de doentes. Para cada cluster de doentes podem ser propostas diferentes intervenções farmacêuticas de acordo com a perceção do risco associado. Por exemplo, dois clusters (2 e 3), representando $36,3 \%$ dos doentes, poderiam beneficiar de uma revisão do tipo $2 \mathrm{~A}$, enquanto outro cluster (4), compreendendo $30,9 \%$ dos doentes, 
Table 3 - Clusters identified and their characteristics $(n=55)$

Tabela 3 - Clusters identificados e suas características $(\mathrm{n}=55)$

\begin{tabular}{|c|c|c|c|}
\hline Cluster & N (\%) & Criteria & Critérios \\
\hline 1 & $17(30.9)$ & $\begin{array}{ll}\text { - } & \text { Minor interactions (100\%) } \\
\text { - } & \text { Medical condition with measurable biomarkers (100\%) } \\
\text { - } & \text { Medicines used (Median; [IQR]): 5.0; [4.0-6.0] } \\
& \text { Beers Criteria: Not applicable (41.2\%); appropriate } \\
& (35.3 \%) ; \text { inappropriate }(23.5 \%) \\
\text { - } & \text { No contraindications }(70.6 \%) \text {; minor }(23.5 \%) \text {; moderate } \\
(5.9 \%)\end{array}$ & $\begin{array}{ll}\cdot & \text { Interações ligeiras }(100 \%) \\
\text { Condição médica com biomarcadores mensuráveis } \\
\\
(100 \%) \\
\text { Medicamentos usados (mediana; [IQR]): 5,0; }[4,0-6,0] \\
\text { - } \quad \text { Critérios de Beers: Não aplicável }(41,2 \%) ; \text { apropriado } \\
& (35,3 \%) ; \text { impróprio }(23,5 \%) \\
& \text { Sem contraindicações }(70,6 \%) ; \text { ligeiras }(23,5 \%) ; \\
& \text { moderadas }(5,9 \%) \\
\end{array}$ \\
\hline 2 & $12(21.8)$ & $\begin{array}{ll}\text { - } & \text { Moderate interactions }(100 \%) \\
. & \text { Medical condition with measurable biomarkers }(100 \%) \\
\text { - } & \text { Medicines used (Median; [IQR]): 8.0; [5.0-10.0] } \\
& \text { Beers Criteria - appropriate (58.3\%); Inappropriate } \\
& (41.7 \%) \\
\text { - } & \text { No contraindications }(50.0 \%) \text {; minor }(16.7 \%) \text {; moderate } \\
(33.3 \%)\end{array}$ & $\begin{array}{ll}\cdot & \text { Interações moderadas }(100 \%) \\
\text { Condição médica com biomarcadores mensuráveis } \\
(100 \%) \\
\text { Medicamentos usados (mediana; [IQR]): 8,0; [5,0-10,0] } \\
\text { Critérios de Beers - apropriado (58,3\%); Inapropriado } \\
(41,7 \%) \\
\text { Sem contraindicações }(50,0 \%) ; \text { ligeiras }(16,7 \%) ; \\
\text { moderadas }(33,3 \%)\end{array}$ \\
\hline 3 & $8(14.5)$ & $\begin{array}{ll}\cdot & \text { No interactions (25.0\%); minor (25.0\%); moderate } \\
& (50.0 \%) \\
& \text { Medical condition without measurable biomarkers } \\
& (87.5 \%) \text {; with measurable biomarkers }(12.5 \%) \\
\text {. } & \text { Medicines used (Median; [IQR]): } 4.0 ;[3.0-6.5] \\
\text { - } & \text { Beers Criteria: not applicable }(100 \%) \\
\text {. } & \text { No contraindications }(87.5 \%) ; \text { moderate }(12.5 \%) \\
\end{array}$ & $\begin{array}{ll}\cdot & \text { Sem interações }(25,0 \%) \text {; ligeiras }(25,0 \%) \text {; moderadas } \\
& (50,0 \%) \\
& \text { Condição médica sem biomarcadores mensuráveis } \\
& (87,5 \%) ; \text { com biomarcadores mensuráveis }(12,5 \%) \\
\cdot & \text { Medicamentos usados (mediana; [IQR]): } 4,0 ;[3,0-6,5] \\
\cdot & \text { Critérios de Beers: não aplicável }(100 \%) \\
\text {. } & \text { Sem contraindicações }(87,5 \%) ; \text { moderadas }(12,5 \%) \\
\end{array}$ \\
\hline 4 & $17(30.9)$ & $\begin{array}{ll}\cdot & \text { No interactions }(100 \%) \\
. & \text { Medical condition with measurable biomarkers }(100 \%) \\
\text { - } & \text { Medicines used (Median; [IQR]): 3.0; [2.0-4.0] } \\
& \text { Beers Criteria: not applicable (47.1\%); Appropriate } \\
& (52.9 \%) \\
\text {. } & \text { No contraindication }(94.1 \%) ; \text { minor }(5.9 \%)\end{array}$ & $\begin{array}{ll}\cdot & \text { Sem interações }(100 \%) \\
\cdot & \text { Condição médica com biomarcadores mensuráveis } \\
& (100 \%) \\
\cdot & \text { Medicamentos usados (mediana; }[\mathrm{IQR}]): 3,0 ;[2,0-4,0] \\
\cdot & \text { Critérios de Beers: não aplicável }(47,1 \%) ; \text { Apropriado } \\
& (52,9 \%) \\
& \text { Sem contraindicações }(94,1 \%) ; \text { ligeiras }(5,9 \%) \\
\end{array}$ \\
\hline Outlier & $1(1.8)$ & $\begin{array}{ll}\cdot & \text { Major interactions } \\
\cdot & \text { Medical condition with measurable biomarkers } \\
\cdot & \text { Medicines used: } 7 \\
\text {. } & \text { Beers Criteria: inappropriate } \\
\text { Major contraindications }\end{array}$ & $\begin{array}{ll}\cdot & \text { Interações graves } \\
\cdot & \text { Condição médica com biomarcadores mensuráveis } \\
\cdot & \text { Medicamentos usados: } 7 \\
\cdot & \text { Critérios de Beers: Inapropriado } \\
\text {. } & \text { Contraindicações graves }\end{array}$ \\
\hline
\end{tabular}

Assessment of interactions Avaliação das interacçõe

Pathology with measurable parameters Patologia com parâmetros mensurávei

Total of medicines used by the patient Total de medicamentos utilizados pelo paciente

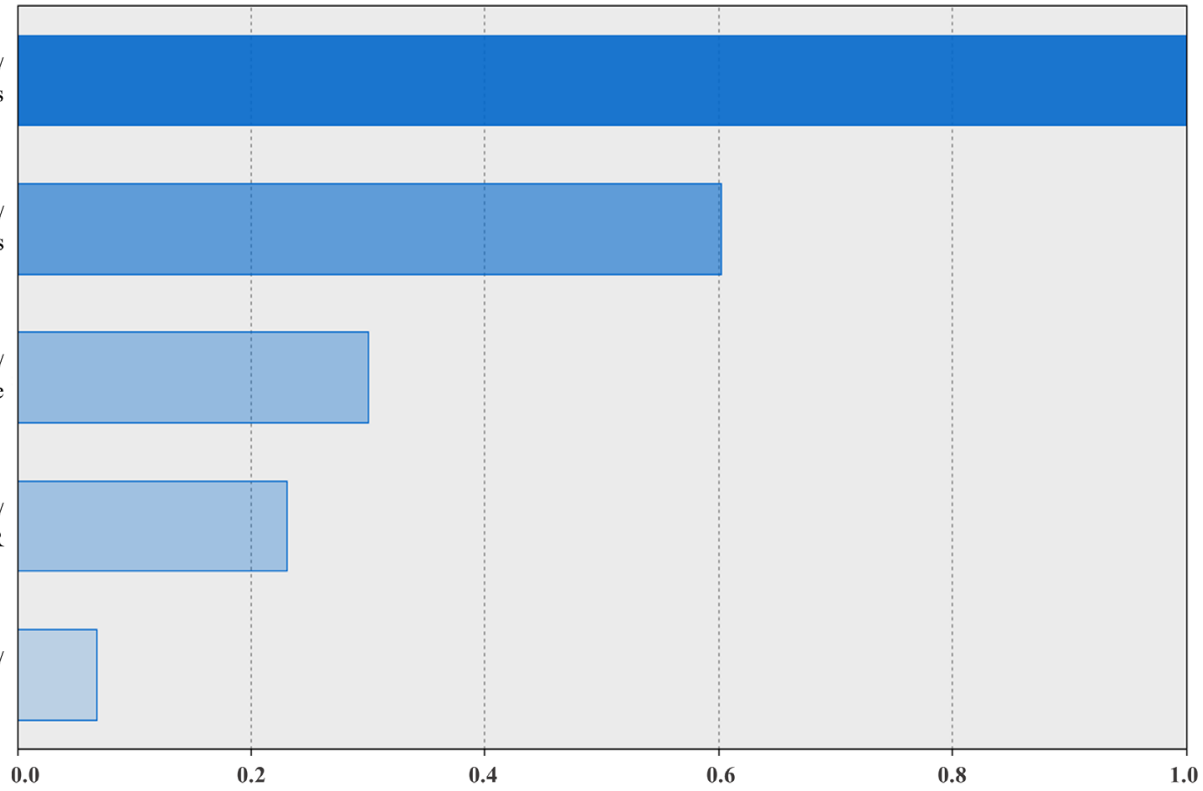

Figure 2 - Variables used in the model and their importance

Figura 2 - Variáveis usadas no modelo e a sua importância 
effectiveness of the therapy. The medication review also identified one high risk patient requiring an urgent type 2A medication review. The results showed that all of the patients in this sample could benefit from professional intervention beyond the basic medication dispensing service. Our analysis showed that while $69 \%$ could benefit from more advanced interventions, simple interventions already integrated in day-to-day pharmacy practice would be suitable for $31 \%$ of patients. These services are low-cost, and have the potential to yield a high degree of patient engagement and satisfaction, critical for a successful longitudinal provision of services (27). The high frequency of visits to the pharmacy ( 1 to 2 times a month), especially in patients who use a higher number of chronic medications, gives community pharmacists the opportunity to provide such professional services without scheduling additional visits. This high frequency is common in the community setting and has been reported in other studies (28-30). Moreover, this high degree of patient-pharmacist interaction represents opportunities to provide additional care. Consistent registration of biomarkers in the patient's electronic record would allow the assessment of the effectiveness of therapies, which our study could not characterize due to the absence of biomarker records. The quality of information held within patient medication records affects the efficiency of new service provided. With more accurate and complete records, the more patientcentered approaches can be taken, with reduced duplication of effort (31).

The main causes of DRP assessed were related to safety - drug interactions, contraindications, and drug appropriateness. DRP identification is one of the first steps in therapeutic optimization, followed by its classification and resolution by the pharmacist or prescriber. As the four cluster model shows, the most important factor in cluster creation was drug interactions. This finding led us to consider the outlier patient as a high risk patient that was undetected at the time of dispensition of medication but in urget need of a type 2A medication review. The use of Sifarma ${ }^{\mathrm{TM}}$ software in the medication review proved to be useful, as it adds a degree of severity to the causes of DRPs, helping to prioritize patients and define the professional intervention of the pharmacists most suited to them. precisaria apenas de medir os seus biomarcadores viabilizando assim a avaliação da efetividade da terapêutica. Além disso, a revisão terapêutica identificou um doente de alto risco, exigindo uma revisão urgente do tipo $2 \mathrm{~A}$. Os resultados mostraram que praticamente todos os doentes desta amostra poderiam beneficiar de outra intervenção profissional além do serviço de dispensa de medicamentos. A nossa análise mostra que cerca de $69 \%$ dos doentes requerem uma intervenção avançada, enquanto $31 \%$ dos doentes seriam elegíveis para intervenções simples já integradas na prática farmacêutica. Esses serviços são de baixo custo, mas têm o potencial de gerar um elevado grau de envolvimento e satisfação do doente, essenciais para uma prestação de serviços longitudinal bem-sucedida (27). A elevada frequência de visitas à farmácia (1 a 2 vezes por mês), principalmente dos doentes que usam um maior número de medicamentos crónicos, dá aos farmacêuticos comunitários a oportunidade de prestar serviços profissionais sem agendamento de consultas adicionais. Essa elevada frequência é comum em ambulatório e também foi encontrada em outros estudos (28-30). Além disso, esse elevado grau de interação farmacêutico-doente possibilita a prestação de cuidados adicionais. $\mathrm{O}$ registo consistente de biomarcadores no registo eletrónico do doente permitiria avaliar a eficácia da terapia, que o nosso estudo não pode caracterizar devido à ausência de registos desses biomarcadores. A qualidade da informação mantida nos registos eletrónicos do doente afeta a eficiência da prestação de novos serviços, pois quanto mais precisos e completos forem, mais abordagens focadas nos doentes podem ser realizadas e com menor duplicação de esforços (31).

As principais causas de PRM avaliadas foram relacionadas com a segurança - interações medicamentosas, contraindicações e adequação dos medicamentos. A identificação de PRM é uma das primeiras etapas na otimização terapêutica seguida da sua classificação e resolução pelo farmacêutico ou prescritor. Como mostra o modelo de quatro clusters, o fator mais importante para decidir a integração em determinado grupo foram as interações medicamentosas. Esta observação levou-nos a considerar o doente outlier como representando doentes de alto risco que não foram sinalizados no momento da dispensa, e que beneficiariam de uma revisão terapêutica tipo $2 \mathrm{~A}$ urgente. $\mathrm{O}$ uso do software Sifarma ${ }^{\mathrm{TM}}$ mostrou-se útil na revisão terapêutica, pois associa o grau de gravidade às causas de PRM, auxiliando na priorização dos doentes e na definição da intervenção profissional da farmácia mais adequada. 
Among the barriers to implementation of new pharmacy services thoroughly identified in the literature, lack of time is constantly cited as one of the most important $(11,12)$. To overcome this barrier, Information Technologies would be a very welcome tool (13). Hence, the need to use an algorithm to identify patients for advanced interventions, integrating a new customer relationship service in the pharmacy workflow without massive disruption, is evident. Having an automated algorithm able to find such patients that would complement current information systems would be an obvious benefit for any pharmacy and pharmacist. In order to help pharmacists in the provision of medication reviews without increasing workload, full automation of type 1 medication review is desirable. This non-clinical review addresses simple issues related to the medication to highlight more complex issues requiring other types of reviews or services. A computerised system based on an algorithm might support a less time-consuming, continuous, and reproducible type 1 medication review (18). The automation of a type 1 medication review involves screening the pharmacy database (from Sifarma ${ }^{\mathrm{TM}}$ ), identifying groups of patients based on predefined criteria, and the suggestion of a professional service. Nevertheless, the full automation of all types of medication reviews cannot be foreseen. No automation replaces pharmacist's assessment. However, accessibility to well-kept electronic patient records is essential for the use of queries and a starting point for criteria-based algorithms (18). Machine Learning, and its ability to use algorithms capturing complex, nonlinear relationships in data, could then make this goal more feasible and efficient (13). We consider that this study was a step in this direction. Future studies on this topic should focus on testing an automated algorithm based on the criteria here identified, assessing its validity, pertinence, and impact on the pharmacy practice. Its use in pharmacy daily practice will enable continuous improvements and more accurate outcome measurement.

This study presents some limitations that must be acknowledged. In spite of the low number of patients in the sample, we consider that it is relevant to show the potential use of the information and data available in the pharmacy's electronic records, since this information is collected consistently over time but remains untapped. However, the low number of patients in the sample hinders the extrapolation of the medication review results to the general population. The frequency and degree of contraindications and interactions are not generalizable. Moreover, most of the drugs targeted for review were solid oral dosage forms, but it is also
De entre as barreiras para implantação de novos serviços de farmácia amplamente identificadas na literatura, a falta de tempo é constantemente citada como uma das mais importantes $(11,12)$. Para superar essa barreira, as tecnologias de informação serão uma ferramenta muito bem-vinda (13). Assim, consideramos evidente a necessidade de utilizar um algoritmo para identificar doentes para intervenções avançadas, integrando assim um novo serviço de relação com o cliente no fluxo de trabalho da farmácia sem grande disrupção. Dispor de um algoritmo automatizado capaz de sinalizar os doentes, complementando o sistema informático atual, constitui uma vantagem óbvia para qualquer farmácia e farmacêutico. A fim de ajudar os farmacêuticos na prestação de serviços sem aumentar a carga de trabalho, é desejável a automatização da revisão terapêutica do tipo 1. Esta revisão, não clínica, aborda questões simples relacionadas com a medicação evidenciando questões mais complexas para outro tipo de revisão ou serviço. Já existe evidência que um algoritmo informatizado pode suportar revisões terapêuticas menos demoradas, contínuas e reprodutíveis (18). A automatização da revisão terapêutica do tipo 1 envolve a triagem da base de dados da farmácia (Sifarma ${ }^{\mathrm{TM}}$ ), a identificação de grupos de doentes com base em critérios pré-definidos e a sugestão de um serviço profissional. No entanto, não é previsível a automatização de todos os tipos de revisões terapêuticas. Nenhuma automatização substitui a avaliação do farmacêutico. Já a acessibilidade aos registos eletrónicos dos doentes é essencial para o uso de queries e um ponto de partida para algoritmos baseados em critérios (21). Dessa forma, o recurso ao Machine Learning com a sua capacidade de usar algoritmos que capturam relações complexas e não lineares em bases de dados, pode viabilizar esse objetivo (13). Consideramos que este estudo foi um passo nessa direcção. Estudos futuros sobre este tópico devem concentrar-se em testar um algoritmo automatizado baseado nos critérios aqui identificados, avaliando a sua validade, pertinência e impacto na prática farmacêutica. A sua utilização na prática diária da farmácia permitirá melhorias contínuas e uma medição mais precisa dos resultados.

Este estudo apresenta algumas limitações que devem ser consideradas. Apesar do baixo número de doentes na amostra, consideramos relevante mostrar o potencial de utilização da informação e dados disponíveis nos registos eletrónicos da base de dados da farmácia, uma vez que essas informações são recolhidas de forma consistente ao longo do tempo, mas permanecem inexploradas Contudo, o baixo número de doentes na amostra dificulta a extrapolação dos resultados da revisão de medicamentos para a população em geral. 
necessary to pay attention to non-solid dosage forms. This may have an impact in determining the severity of interactions, since the software only identifies the drug interaction, leaving the assessment of its importance to the pharmacist. In addition, the identification of potentially inappropriate medications would have been more complete if there had been access to the respective patient's diagnosis, as has been noted in a previous study (25). Nevertheless, Lavrador et al. (25) found that although restricted access to patients' diagnoses may limit the judgement of Beers PIM-qualifying criteria, this limitation had no effect on the number of PIM identified. This finding confirms that the identification of PIM even without diagnostic information is feasible and should be performed.

The definition of clusters using two-step cluster analyses can also be questioned. Although this method is essentially descriptive and therefore adapted to the exploratory analysis here reported, part of the assumptions for its validity, namely the normal distribution of continuous variables, were not present. However, as the variables included were independent from each other, the categorical variables had a multinomial distribution, and there was a low multicollinearity degree assessed by the VIF, it is possible that, even if all the assumptions are not fully met, the resulting clusters are still valid (32). As the objective was to identify clusters in this sample, the results presented here can only be applied to the pharmacy where the study took place. The rational for attributing pharmaceutical services to each cluster is also open for debate. Our choice was determined by our view of the medication review process, where type 1 medication review implies the identification of DRPs and making recommendations. The screening of the pharmacy database, the criteria that allow signalling the patients, an output that identifies the patient, the reasons for the signalling and the suggestion / recommendation of a professional service are the necessary cornerstones of a future intervention. Based on this output, the pharmacist must assess the situation, schedule the service and provide it, registering new information that will then feed into the algorithm, providing the basis for a "roundabout" strategy for customer loyalty as well as a contribution to the sustainability of the pharmacy. Future medication reviews using an automated algorithm in a larger set of pharmacies with a higher number of patients should validate the identified clusters.
A frequência e o grau de contraindicações e interações não são generalizáveis. Além disso, a maioria dos medicamentos alvo de revisão terapêutica eram formas farmacêuticas orais sólidas, mas também é necessário prestar atenção às formas farmacêuticas não sólidas. Isso pode ter impacto na determinação da gravidade das interações, uma vez que o software apenas identifica a interação medicamentosa, cabendo ao farmacêutico a avaliação da sua importância. No que respeita a identificação de medicamentos potencialmente inadequados (MPI) teria sido mais completa se houvesse acesso ao respetivo diagnóstico do doente, conforme citado em estudo anterior (25). No entanto, Lavrador et al. (25) verificaram que, embora a falta de acesso aos diagnósticos dos doentes possa limitar a aplicação dos critérios de qualificação de Beers, esta limitação não teve efeito no número de MPI identificados. Este resultado confirma que a identificação de MPI, mesmo sem diagnóstico, é viável e deve ser realizada.

A definição de clusters por meio da análise de clusters em duas fases pode também ser questionada. Apesar deste método ser essencialmente descritivo e por isso adaptado à análise exploratória aqui reportada, parte dos pressupostos para a sua validade, nomeadamente a distribuição normal das variáveis contínuas, não foram observados. Contudo, como as variáveis incluídas eram independentes, as variáveis categóricas tinham uma distribuição multinominal e houve um baixo grau de multicolinearidade avaliado pelo VIF, é possível que, mesmo que todos os pressupostos não sejam completamente cumpridos, os clusters resultantes continuem a ser válidos (32). Como o objetivo foi identificar clusters nesta amostra, os resultados aqui apresentados só podem ser aplicados na farmácia onde ocorreu o estudo. De igual forma, o racional para atribuir serviços farmacêuticos a cada cluster está aberta a debate. A sugestão de serviços apresentada foi determinada pela nossa visão do processo de revisão terapêutica, onde a revisão do tipo 1 implica a identificação de PRM, ou causas de PRM, e fazer recomendações que permitam a sua resolução. A triagem da base de dados da farmácia, os critérios que permitem sinalizar os doentes, um output que identifica o doente, os motivos da sinalização e a sugestão / recomendação de um serviço profissional são os pilares necessários para uma futura intervenção farmacêutica. Com base neste output, o farmacêutico deve avaliar a situação, agendar o serviço e prestá-lo, registando nova informação que alimenta o algoritmo, fornecendo a base para uma estratégia de "carrossel" (i.e., ciclo contínuo de prestação de serviços farmacêuticos de acordo com as necessidades) para fidelizar o cliente e 


\section{Conclusions}

Current pharmacy practice generates a wealth of information regarding patients that is underused. To provide more and better professional services, community pharmacists need tools and systems that can analyse this information, processing data easily, quickly, and continuously.

The type 1 medication review conducted in the patients' electronic records of a pharmacy, using the Sifarma ${ }^{\mathrm{TM}}$ system, alerted to drug interactions and contraindications that, combined with the Beers criteria, allowed the identification of potential DRPs. The statistical analysis then allowed the grouping of patients into clusters, enabling their prioritization and subsequent suggestions of pharmaceutical interventions according to their health needs.

With current IT technology, we were able to identify patients who could benefit from professional pharmaceutical services, select the most appropriate intervention, and suggest their scheduling according to the frequency of the patients' visits to the pharmacy. Further studies will include the testing of a new IT tool to allow continuous screening of the pharmacy database to identify patients at risk who will benefit most from the professional services of the pharmacy. With an algorithm-based system and new pharmacy workflows, the ubiquitous barrier of lack of time can be overcome, enhancing the community pharmacist's ability to contribute to the quality use of medications.

\section{Authors Contributions Statement}

LR, conceptualization and study design; MM and LL, experimental implementation; LR, MM, and JG, data analysis; LR and JG, drafting, editing and reviewing; LR, figures and graphics; JG, supervision and final writing. também contribuir para sustentabilidade da farmácia. Futuras revisões terapêuticas usando um algoritmo automatizado num conjunto alargado de farmácias com um maior número de doentes permitirão validar os clusters identificados.

\section{Conclusões}

A Farmácia de hoje tem uma riqueza de informação sobre seus utentes que está subutilizada. Para fornecer mais e melhores serviços profissionais, os farmacêuticos comunitários precisam de ferramentas e sistemas que analisem esses dados e processem a informação de forma fácil, rápida e contínua.

A revisão da medicação tipo 1 realizada nos registos eletrónicos dos doentes da farmácia usando o sistema Sifarma $^{\mathrm{TM}}$ alertou para interações de medicamentos e contraindicações que conjugadas com os critérios de Beers, permitiu identificar potenciais PRM. A análise estatística realizada permitiu o agrupamento dos doentes em clusters, possibilitando a sua priorização e posterior definição de intervenções farmacêuticas em função das suas necessidades de saúde.

Com as tecnologias de informação atuais, pudemos identificar os doentes que podem beneficiar de serviços profissionais da farmácia, selecionar a intervenção mais adequada e sugerir o seu agendamento de acordo com a frequência de visita do doente à farmácia. Os próximos desenvolvimentos consistirão em testar uma nova ferramenta informática que permita triagem contínua da base de dados da farmácia com a identificação dos doentes em risco que mais beneficiarão dos serviços profissionais da farmácia. Com um sistema baseado num algoritmo e novos fluxos de trabalho na farmácia, a barreira omnipresente da falta de tempo poderá ser superada, aumentando a capacidade de o farmacêutico comunitário contribuir para o uso correto, eficaz e seguro de medicamentos.

\section{Declaração sobre as contribuições do autor}

LR, conceção e desenho do estudo; MM e LL, implementação experimental; LR, MM e JG, análise de dados; LR e JG, redação, edição e revisão; LR, figuras e gráficos; JG, supervisão e redação final. 


\section{Funding}

This study was supported by Farmácia Central do Cacém, Lda., and funded by national funds through FCT - Foundation for Science and Technology, I.P., under the UIDB/04567/2020 and UIDP/ 04567/2020 projects. João Gregório is funded by Foundation for Science and Technology (FCT) Scientific Employment Stimulus contract with the reference number CEEC/ CBIOS/EPH/2018

\section{Acknowledgements}

The authors would like to express their thanks Farmácia Central do Cacém, Lda.

\section{Conflict of Interests}

The authors declare there are no financial and/or personal relationships that could present a potential conflict of interests.

\section{Financiamento}

Este estudo foi apoiado pela Farmácia Central do Cacém, Lda. e financiado por fundos nacionais através da FCT - Fundação para a Ciência e Tecnologia, I.P., no âmbito dos projetos UIDB / 04567/2020 e UIDP / 04567/2020. João Gregório é financiado pelo contrato de Estímulo ao Emprego Científico da Fundação para a Ciência e Tecnologia (FCT) com o número de referência CEEC / CBIOS / EPH / 2018

\section{Agradecimentos}

Os autores desejam expressar os seus agradecimentos à Farmácia Central do Cacém, Lda.

\section{Conflito de Interesses}

Os autores declaram que não há relações financeiras e/ou pessoais que possam representar um potencial conflito de interesses. 


\section{References / Referências}

1. Meraya, A. M., Raval, A. D., \& Sambamoorthi, U. (2015). Chronic condition combinations and health care expenditures and out-of-pocket spending burden among adults, Medical Expenditure Panel Survey, 2009 and 2011. Preventing Chronic Disease, 12, E12. https://doi.org/10.5888/pcd12.140388.

2. Bodenheimer, T., Wagner, E. H., \& Grumbach, K. (2002). Improving primary care for patients with chronic illness. JAMA, 288(14), 1775-1779. https:// doi.org/10.1001/jama.288.14.1775.

3. Makowsky, M. J., Schindel, T. J., Rosenthal, M., Campbell, K., Tsuyuki, R. T., \& Madill, H. M. (2009). Collaboration between pharmacists, physicians and nurse practitioners: a qualitative investigation of working relationships in the inpatient medical setting. Journal of Interprofessional Care, 23(2), 169-184. https://doi.org/10.1080/13561820802602552.

4. Cranor, C. W., Bunting, B. A., \& Christensen, D. B. (2003). The Asheville Project: long-term clinical and economic outcomes of a community pharmacy diabetes care program. Journal of the American Pharmaceutical Association (Washington, D.C. : 1996), 43(2), 173-184. https://doi org/10.1331/108658003321480713.

5. Hatah, E., Braund, R., Tordoff, J., \& Duffull, S. B. (2014). A systematic review and meta-analysis of pharmacist-led fee-for-services medication review. British Journal of Clinical Pharmacology, 77(1), 102-115. https://doi.org/10.1111/bcp.12140.

6. Huiskes, V. J., Burger, D. M., van den Ende, C. H., \& van den Bemt, B. J. (2017). Effectiveness of medication review: a systematic review and metaanalysis of randomized controlled trials. BMC Family Practice, 18(1), 5. https://doi.org/10.1186/s12875-016-0577-x).

7. Pharmaceutical Care Network Europe Foundation (2012). PCNE statement on medication review 2011-2012.

8. Blenkinsopp, A., Bond, C., \& Raynor, D. K. (2012). Medication reviews. British Journal of Clinical Pharmacology, 74(4), 573-580. https://doi. org $/ 10.1111 / \mathrm{j} .1365-2125.2012 .04331 . \mathrm{x})$.

9. Soares, M. A., Fernandez-Llimos, F., Cabrita, J., \& Morais, J. (2011). Critérios de avaliação de prescrição de medicamentos potencialmente inapropriados: uma revisão sistemática [Tools to evaluate potentially inappropriate prescription in the elderly: a systematic review]. Acta Medica Portuguesa, 24(5), $775-784$..

10. By the 2019 American Geriatrics Society Beers Criteria ${ }^{\circledR}$ Update Expert Panel (2019). American Geriatrics Society 2019 Updated AGS Beers Criteria ${ }^{\circledR}$ for Potentially Inappropriate Medication Use in Older Adults. Journal of the American Geriatrics Society, 67(4), 674-694. https://doi.org/10.1111/ jgs. 15767.

11. Niquille, A., Lattmann, C., \& Bugnon, O. (2010). Medication reviews led by community pharmacists in Switzerland: a qualitative survey to evaluate barriers and facilitators. Pharmacy Practice, 8(1), 35-42. https://doi.org/10.4321/s1886-36552010000100004.

12. Coane, S., \& Payne, R. (2016) Carrying out a structured medication review. Prescriber 27, 22-26. https://doi.org/10.1002/psb.1426

13. Gregório, J., \& Cavaco, A. (2021). The pharmacist's guide to the future: Are we there yet?. Research in Social \& Administrative Pharmacy : RSAP, 17(4), 795-798. https://doi.org/10.1016/j.sapharm.2020.05.029

14. Baines, D., Nørgaard, L. S., Babar, Z. U., \& Rossing, C. (2020). The Fourth Industrial Revolution: Will it change pharmacy practice?. Research in Social \& Administrative Pharmacy : RSAP, 16(9), 1279-1281. https://doi.org/10.1016/j.sapharm.2019.04.003).

15. Gulavani, S. S., \& Kulkarni, R. V. (2010) Role of information technology in health care. Proceedings of the 4th National Conference; INDIACom-2010.

16. Jackson, S., \& Peterson, G. (2019). My Health Record: a community pharmacy perspective. Australian Prescriber, 42(2), 46-47. https://doi.org/10.18773/ austprescr.2019.009

17. van Boven, J. F., Stuurman-Bieze, A. G., Hiddink, E. G., Postma, M. J., \& Vegter, S. (2014). Medication monitoring and optimization: a targeted pharmacist program for effective and cost-effective improvement of chronic therapy adherence. Journal of Managed Care \& Specialty Pharmacy, 20(8), 786-792. https://doi.org/10.18553/jmcp.2014.20.8.786.

18. de Wit, H. A., Mestres Gonzalvo, C., Hurkens, K. P., Mulder, W. J., Janknegt, R., Verhey, F. R., Schols, J. M., \& van der Kuy, P. H. (2013). Development of a computer system to support medication reviews in nursing homes. International Journal of Clinical Pharmacy, 35(5), 668-672. https://doi. org/10.1007/s11096-013-9827-3.

19. Roten, I., Marty, S., \& Beney, J. (2010). Electronic screening of medical records to detect inpatients at risk of drug-related problems. Pharmacy World \& Science : PWS, 32(1), 103-107. https://doi.org/10.1007/s11096-009-9352-6.

20. Poudel, A., Ballokova, A., Hubbard, R. E., Gray, L. C., Mitchell, C. A., Nissen, L. M., \& Scott, I. A. (2016). Algorithm of medication review in frail older people: Focus on minimizing the use of high-risk medications. Geriatrics \& Gerontology International, 16(9), 1002-1013. https://doi.org/10.1111/ ggi.12589).

21. Thiruchelvam, K., Wong, P. S., Kairuz, T., Babar, Z. U., \& Hasan, S. S. (2018). Consolidated Medication Review Algorithm to Improve Medications Use in Older Adults: Components, Scoring Scheme, and Implementation. Journal of the American Medical Directors Association, 19(8), 717-718. https:// doi.org/10.1016/j.jamda.2018.03.007).

22. Grupo de Investigación en Atención Farmacéutica (CTS131). Universidad de Granada; Grupo de Investigación en Farmacología Aplicada y Farmacoterapia (CTS259). Universidad de Sevilla; Grupo de Investigación en Farmacología (CTS164). Universidad de Granada (2002) Segundo consenso de Granada sobre problemas relacionados con medicamentos. Ars Pharmaceutica, 43(3-4): 179-187. http://hdl.handle.net/10481/28237

23. ANF - Sifarma Software. (2019) Manual da Compenente Profissional - Sifarma 2000 Software.

24. By the American Geriatrics Society 2015 Beers Criteria Update Expert Panel (2015). American Geriatrics Society 2015 Updated Beers Criteria for Potentially Inappropriate Medication Use in Older Adults. Journal of the American Geriatrics Society, 63(11), 2227-2246.

25. Lavrador, M., Silva, A. A., Cabral, A. C., Caramona, M. M., Fernandez-Llimos, F., Figueiredo, I. V., \& Castel-Branco, M. M. (2019). Consequences of ignoring patient diagnoses when using the 2015 Updated Beers Criteria. International Journal of Clinical Pharmacy, 41(3), 751-756. https://doi. org/10.1007/s11096-019-00828-0.

26. Masnoon, N., Shakib, S., Kalisch-Ellett, L., \& Caughey, G. E. (2017). What is polypharmacy? A systematic review of definitions. BMC Geriatrics, 17(1), 230. https://doi.org/10.1186/s12877-017-0621-2.

27. Gregório, J., Russo, G., \& Lapão, L. V. (2016). Pharmaceutical services cost analysis using time-driven activity-based costing: A contribution to improve community pharmacies' management. Research in Social \& Administrative Pharmacy : RSAP, 12(3), 475-485. https://doi.org/10.1016/j. sapharm.2015.08.004.

28. Irish Pharmacy Union (2018). Vision for community pharmacy in Ireland. https://ipu.ie/wp-content/uploads/2018/04/PwC_IPU-report.pdf.

29. Boardman, H., Lewis, M., Croft, P., Trinder, P., \& Rajaratnam, G. (2005). Use of community pharmacies: a population-based survey. Journal of Public Health (Oxford, England), 27(3), 254-262. https://doi.org/10.1093/pubmed/fdi032.

30. Gregório, J., Cavaco, A. M., \& Lapão, L. V. (2017). How to best manage time interaction with patients? Community pharmacist workload and service provision analysis. Research in Social \& Administrative Pharmacy : RSAP, 13(1), 133-147. https://doi.org/10.1016/j.sapharm.2016.02.008.

31. Wright, D. J., \& Twigg, M. J. (2016). Community pharmacy: an untapped patient data resource. Integrated Pharmacy Research \& Practice, 5, 19-25. https://doi.org/10.2147/IPRP.S8326

32. IBM. Clustering Principles. (2020) IBM Knowledge Center [www.ibm.com/support/knowledgecenter/pt/SSLVMB_24.0.0/spss/tutorials/twostep_ methodology.html| 